\title{
The front-end electronics of the LHCb Ring-Imaging-Cherenkov system
}

\author{
Ken Wyllie, CERN \\ on behalf of the LHCb RICH Group
}

Introduction to the system

Photon-detector: the pixel HPD

Pixel front-end chip

Bump-bonding

Readout electronics

Conclusions 


\section{LHCb}

\section{LHCb RICH photon-detector system}

Reference: talk by Thierry Gys

4 arrays of HPDs covering $2.6 \mathrm{~m}^{2}$ in total

$\sim 500$ pixel HPDs

$\sim 500,000$ channels of data

HPDs mounted on columns together with readout electronics, power distribution \& active cooling

Each column = one independent module

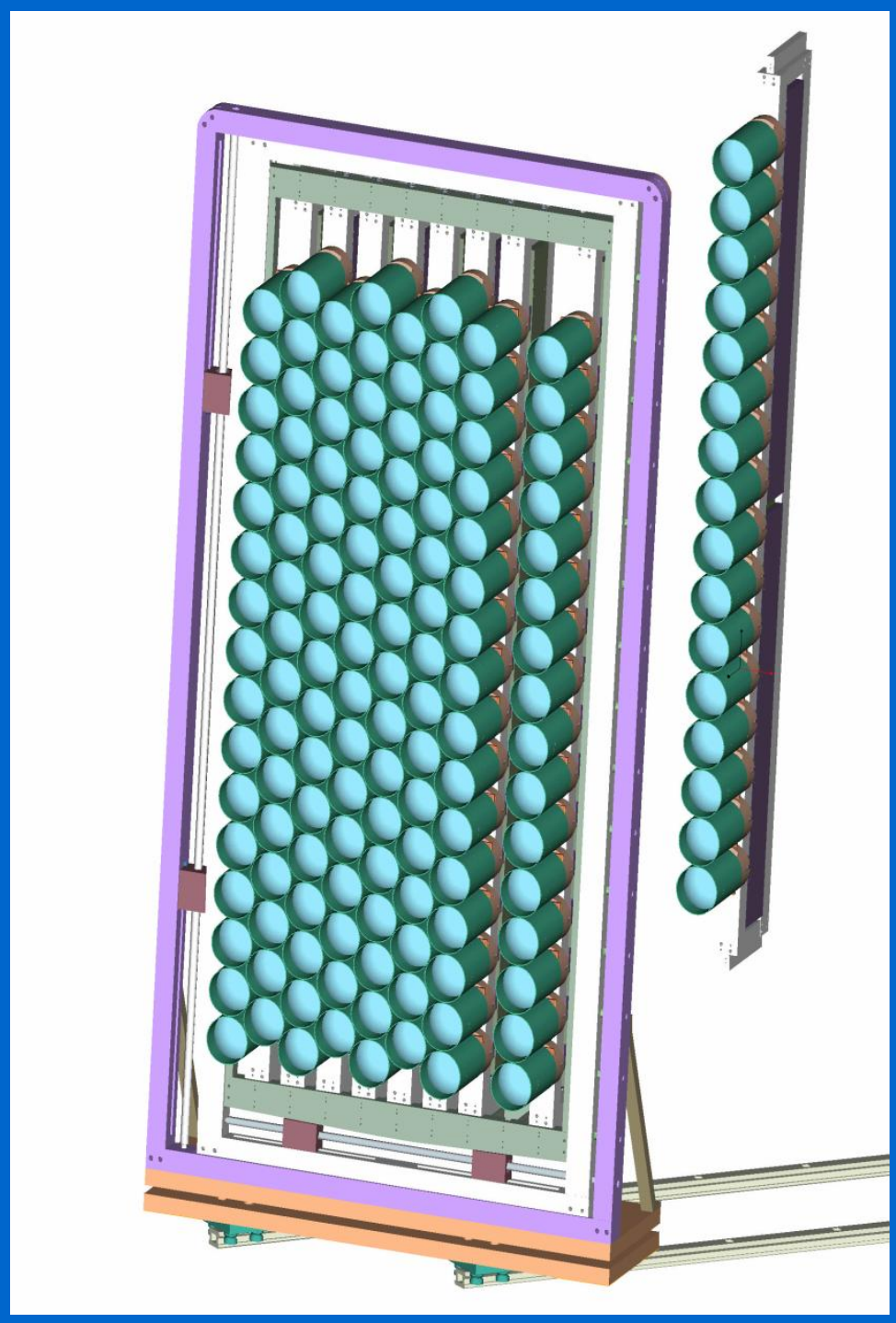


Electronics System

Column

Side-view
On-detector

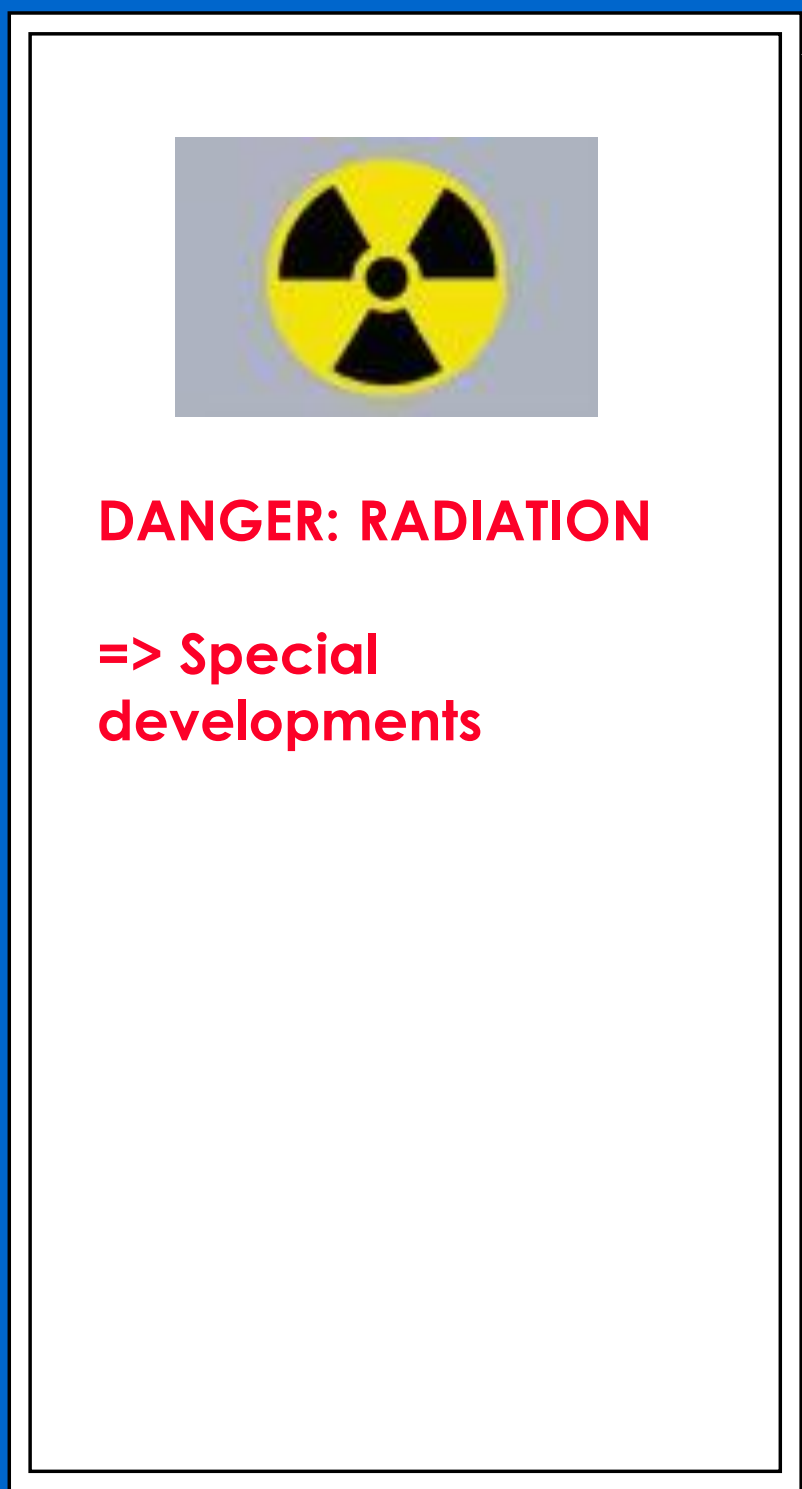

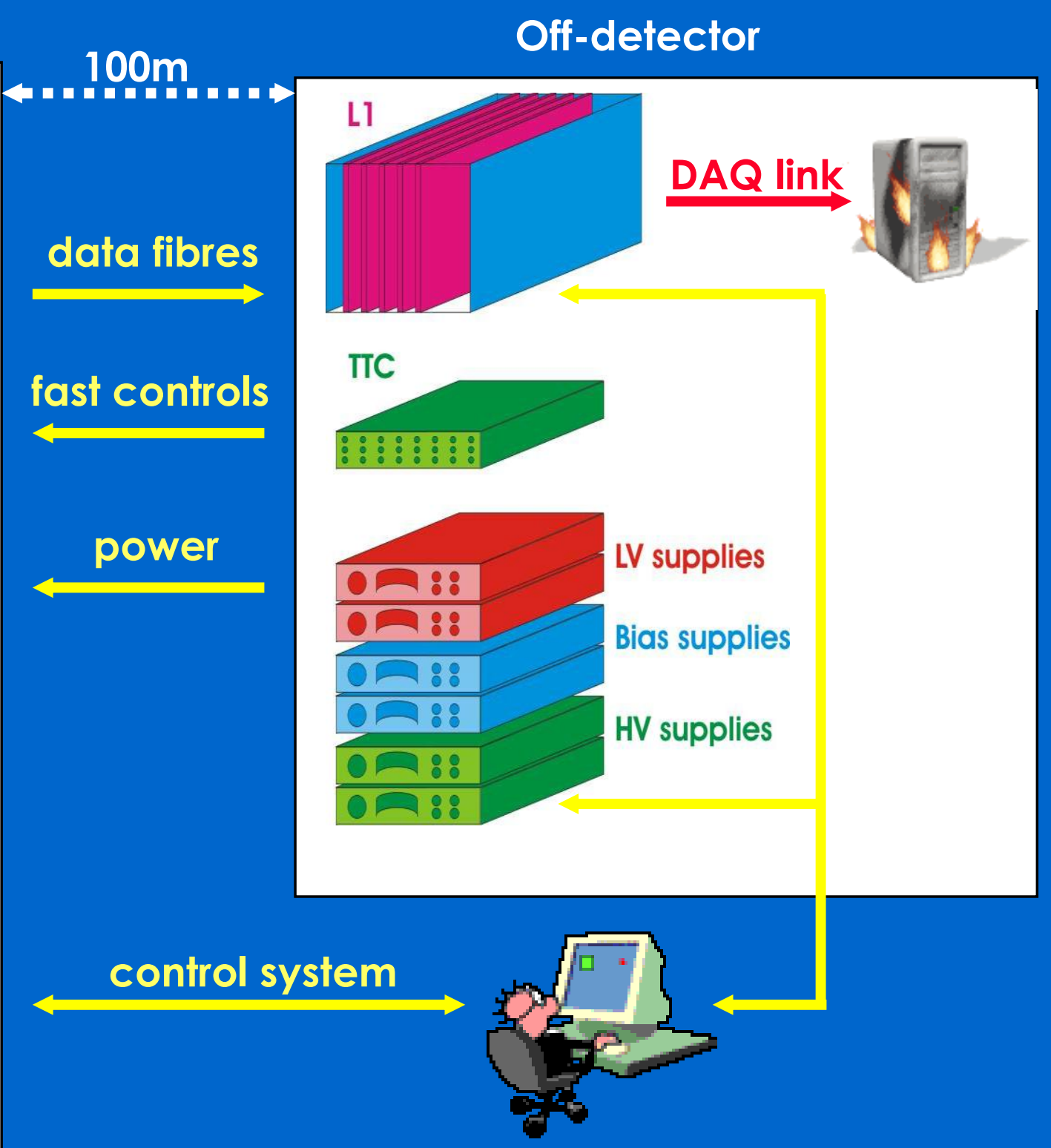

3
Beaune meeting 2005 

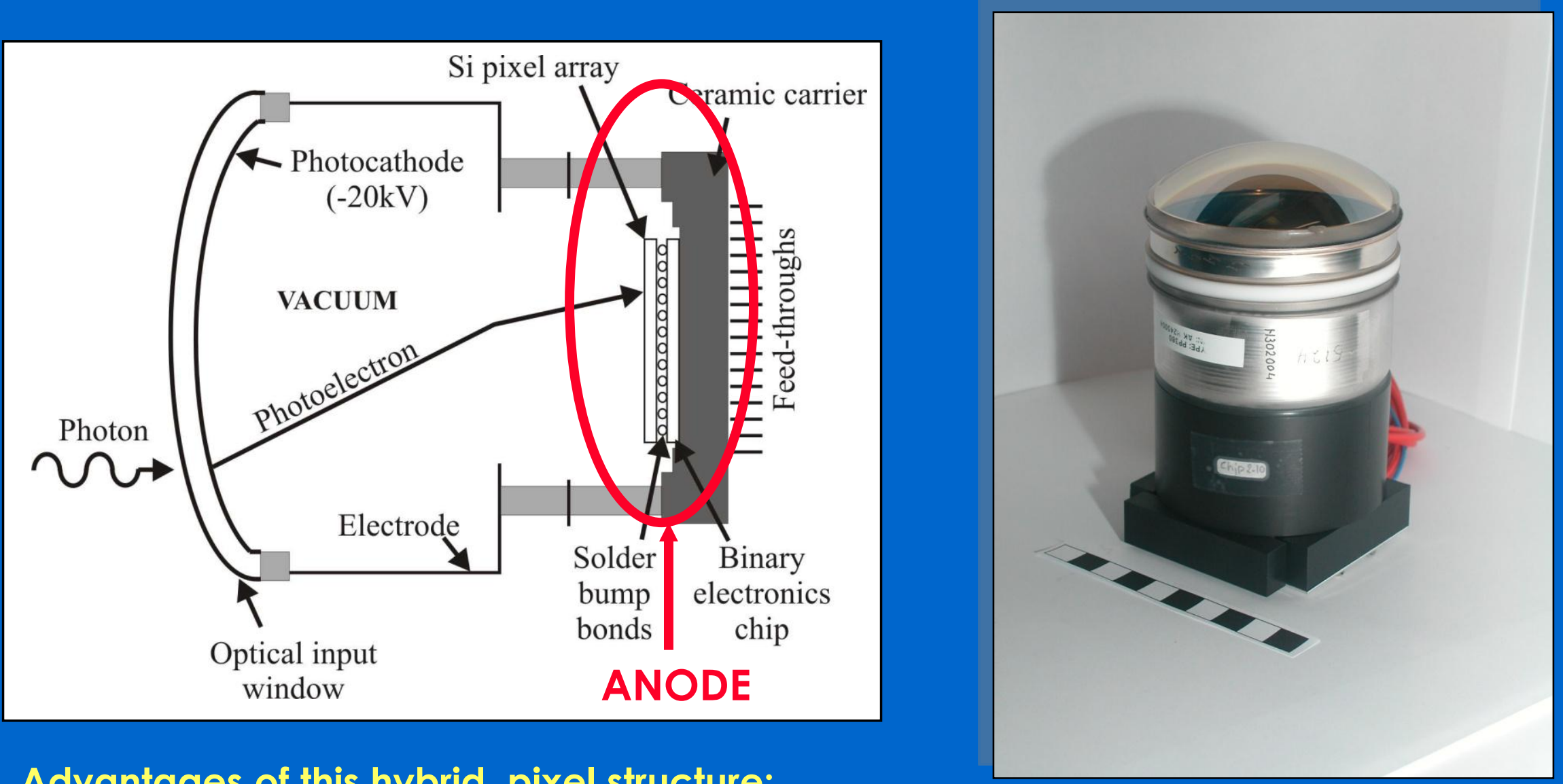

Advantages of this hybrid, pixel structure:

low noise: excellent resolution of single photoelectrons

DEP, The Netherlands high channel number/density 


\section{Flip-chip (bump) bonding}

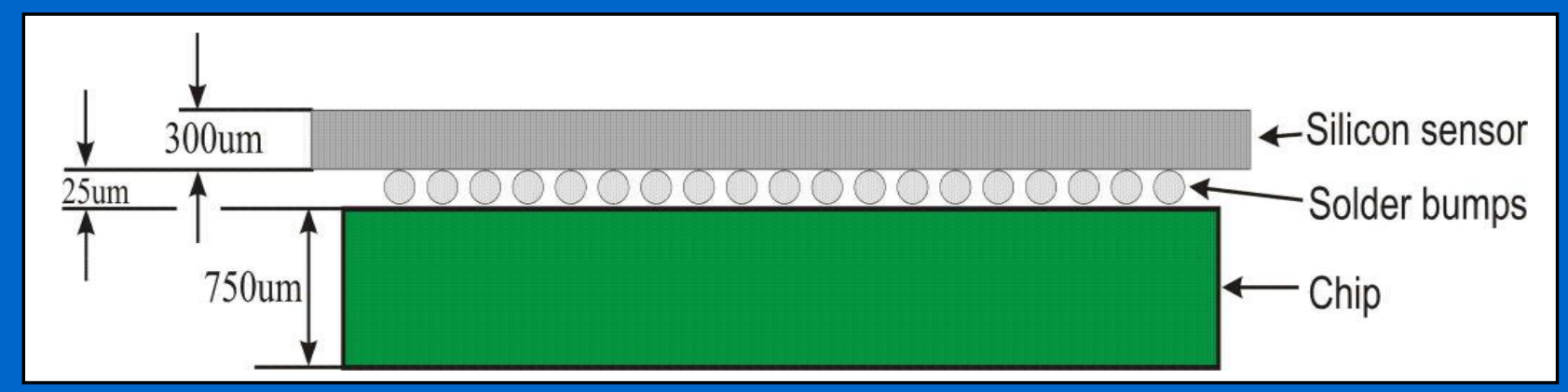

Sensor from Canberra, Belgium, designed specifically for HPD application

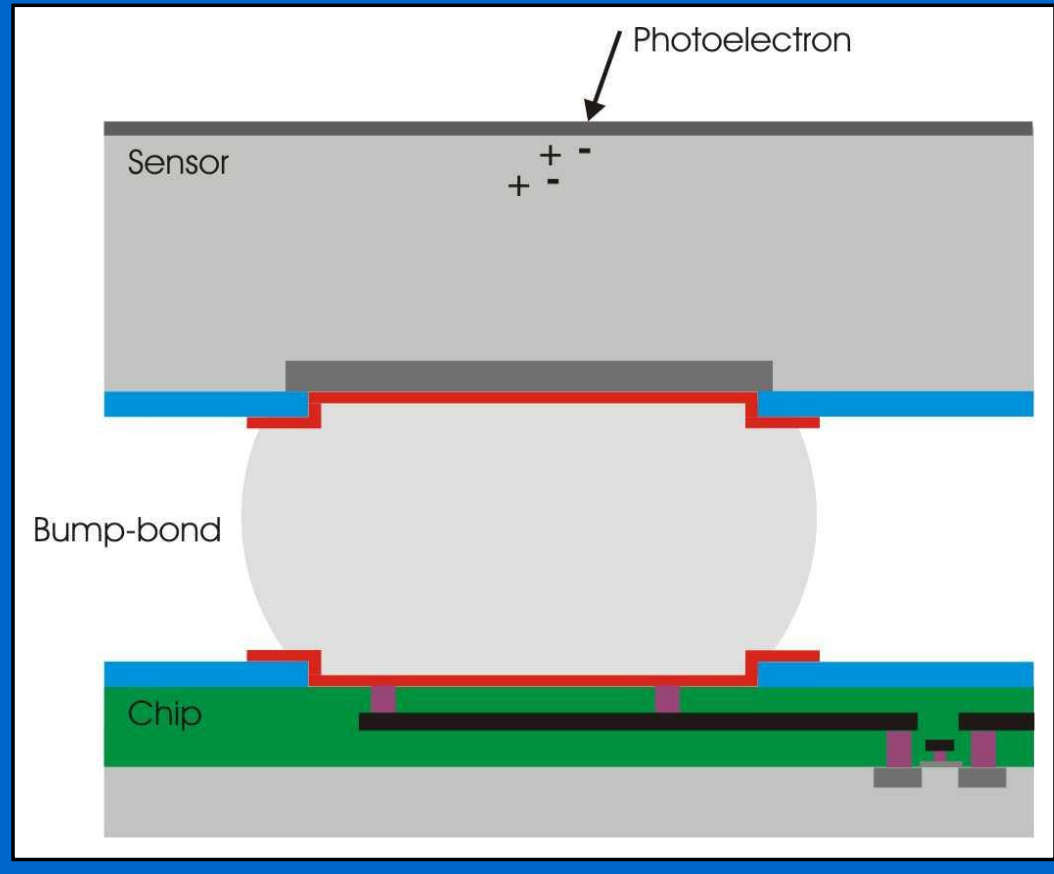

Custom chip fabricated in commercial CMOS
HPD high voltage of $20 \mathrm{kV}$ $\Rightarrow 5000 e^{-}$signal

But.........

- Charge sharing

- Back-scattering

Both reduce signal size 


\section{Pixel chip requirements}

- Low detection threshold $<2000 e^{-}$

- Low noise < $300 e^{-}$

- $\quad 500 \mu \mathrm{m} \times 500 \mu \mathrm{m}$ channel size (focus $\times 5$ )

- $16 \mathrm{~mm} \times 16 \mathrm{~mm}$ active area

- $\quad 25$ ns time precision (LHC) \& fast pulse recovery

- Event rate $\sim 1 \mathrm{MHz}$ ('frame rate')

- Consecutive reading \& writing

- Compatible with HPD manufacturing

Other design issues:

Physics performance not improved by resolving 1 or 2 or 3 photoelectrons

$\Rightarrow \quad$ choice of binary architecture: output $=0$ or 1, threshold applied

$\Rightarrow$ All digital I/Os - try to make it plug-and-play! 


\section{Pixel chip design - LHCBPIX1}

- Commercial $0.25 \mu \mathrm{m}$ CMOS process

- 6 metal layers

- Radiation-tolerant layout

- 13 million transistors

- $1.8 \mathrm{~W}$ total power $(40 \mathrm{MHz}$ clk, $1 \mathrm{MHz}$ trig)

- Digital I/Os

- Internal bias DACs

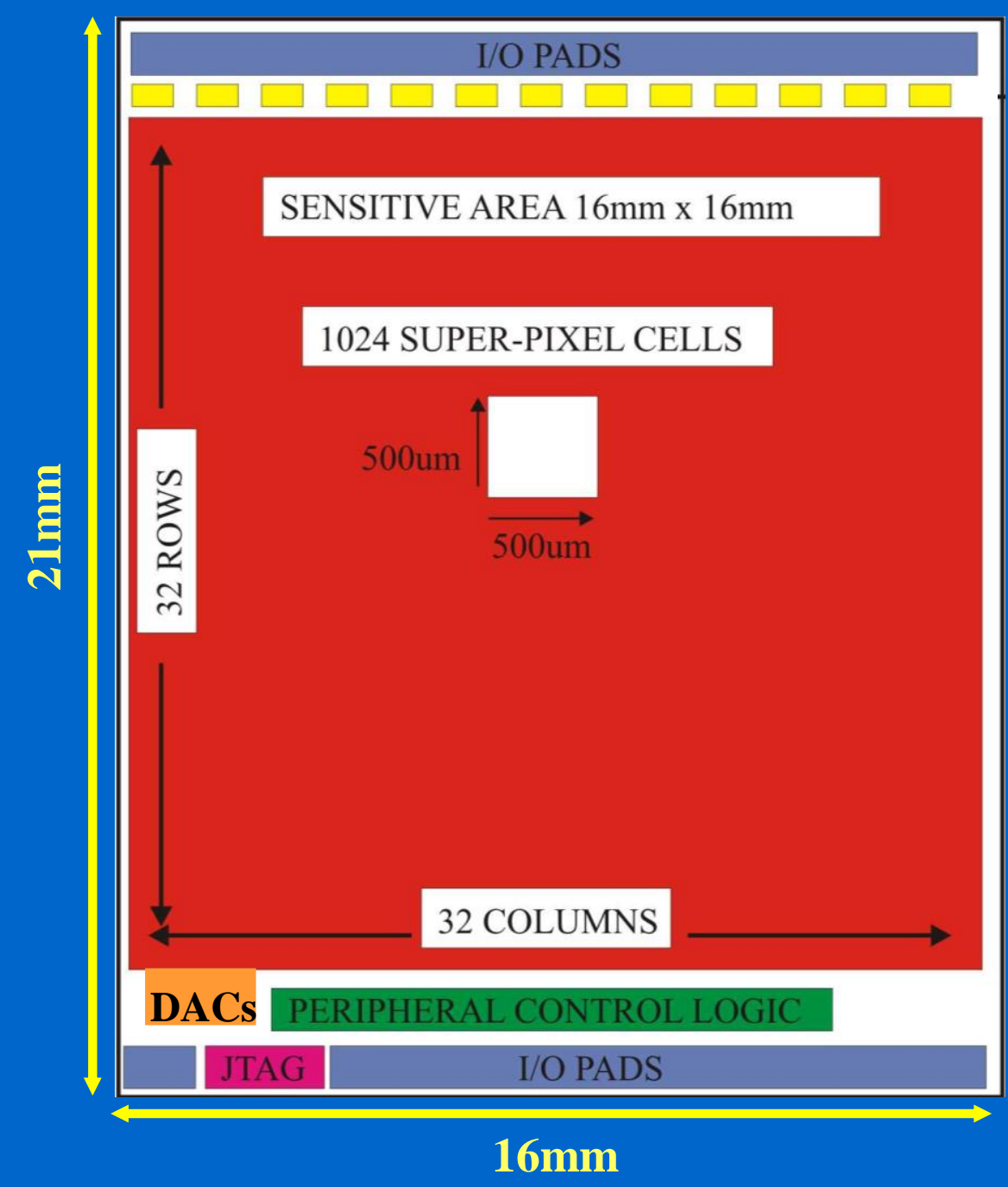

7

Beaune meeting 2005 


\section{Pixel cell}

One super-pixel (500um * 500um) = 8 mini-pixels (62.5um *500um) Internal logic makes a logical $O R$ of hits $\Rightarrow 2$ modes of operation 1024 or 8192 channels

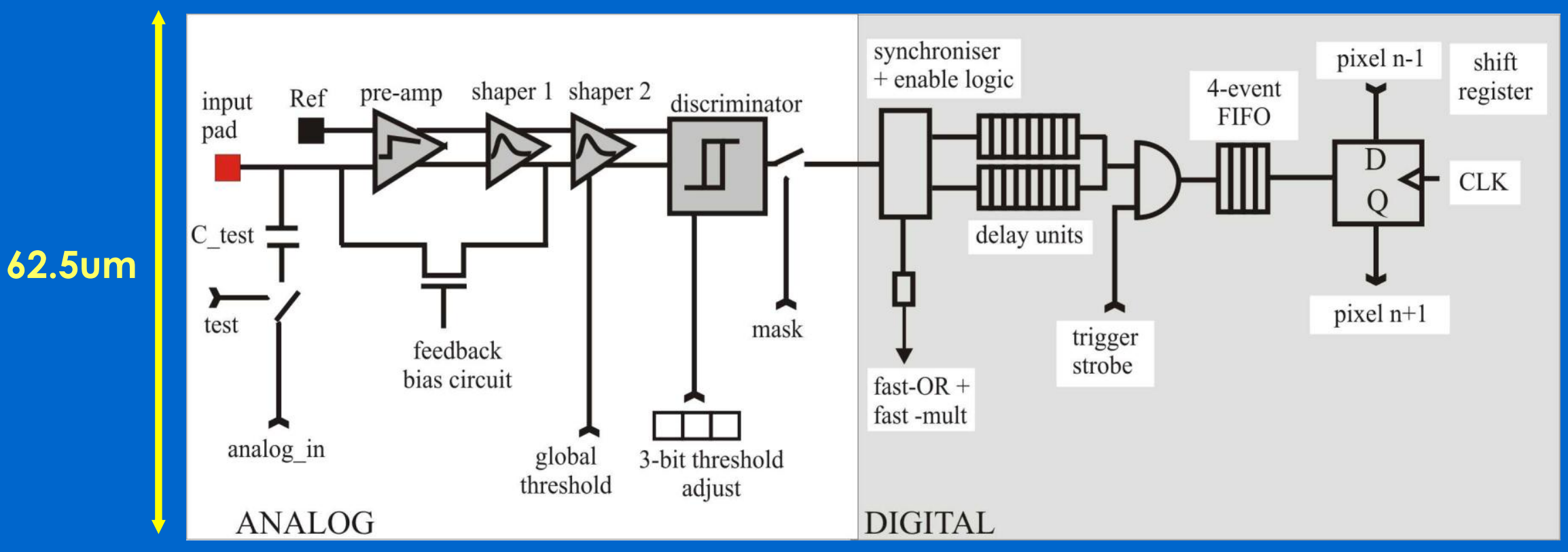

500um

K.Wyllie 


\section{Electrical results - signal sensitiviły}

Discriminator threshold and noise distributions across all pixels

Maximum signal at $20 \mathrm{kV}=5000 \mathrm{e}^{-}$

Charge-sharing \& back-scattering $\Rightarrow>2000 e^{-}$
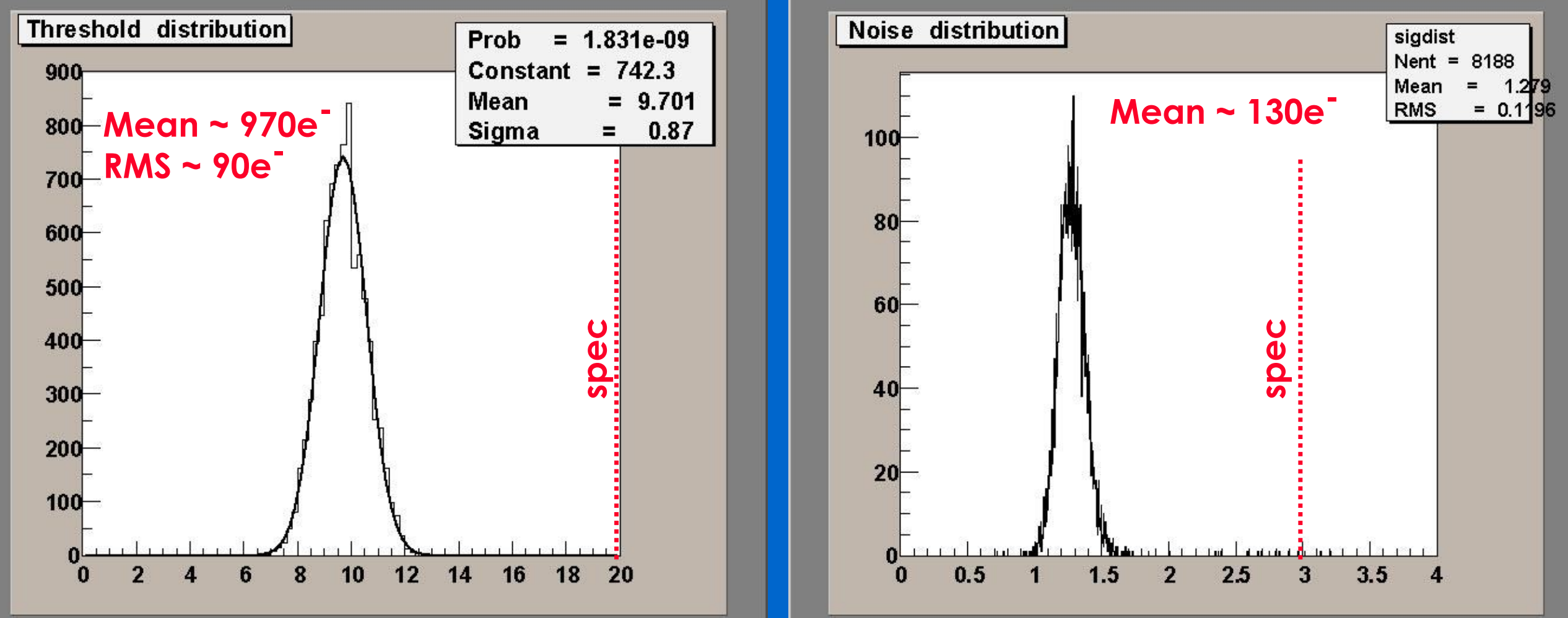

K.Wyllie 


\section{LHCb
THCP}

\section{Electrical results - pulse shaping}

Analog front-end fast recovery $\Rightarrow$ low risk of pile-up

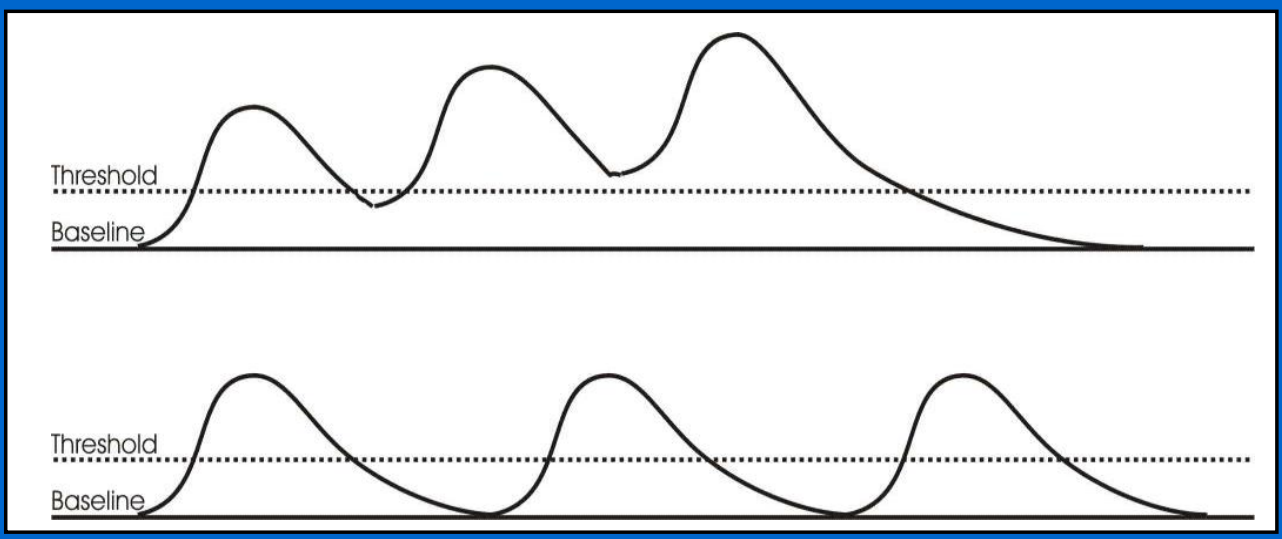

Shaper output

Maximum mean pulse rate per channel $\sim 3 \mathrm{MHz}$

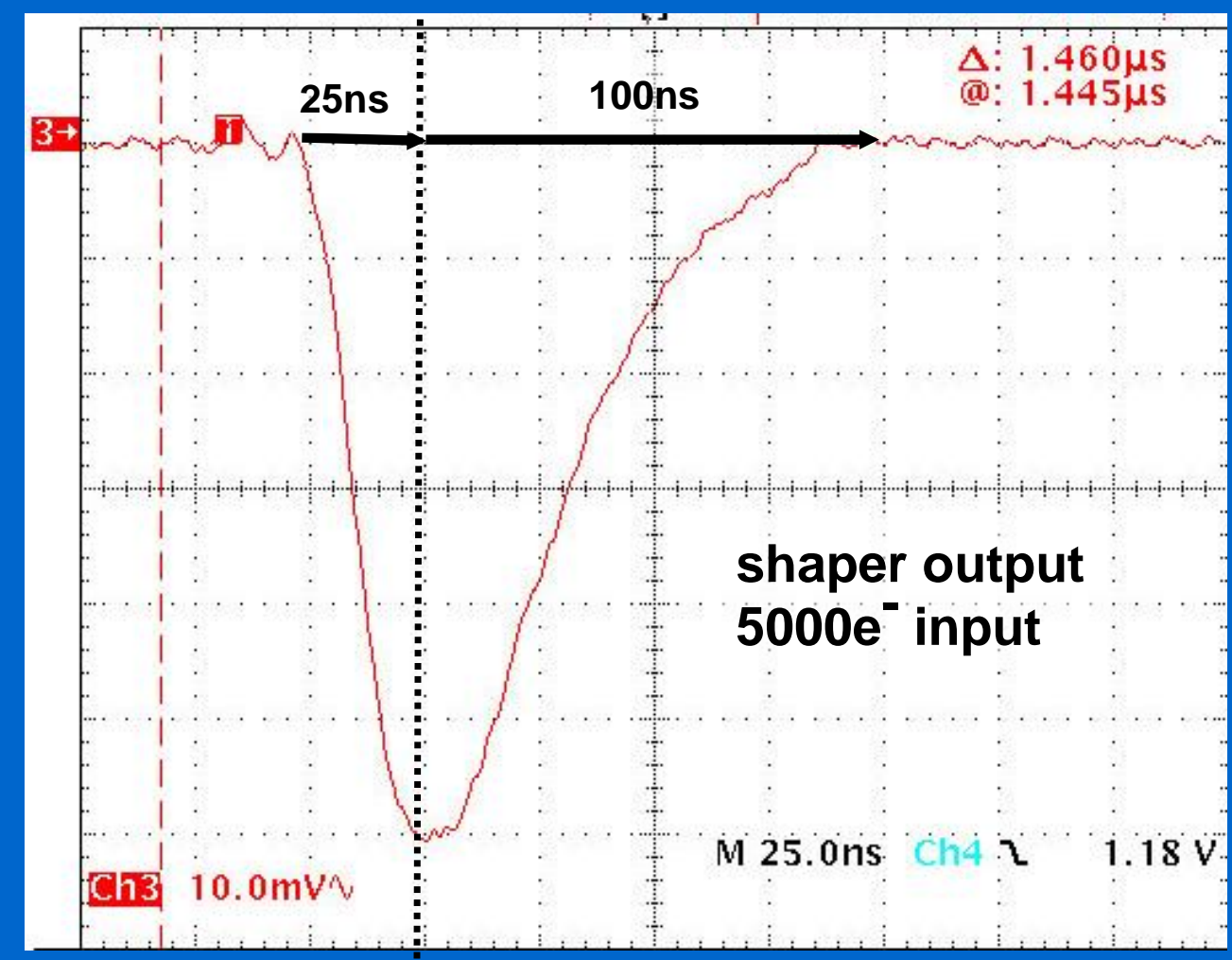

K.Wyllie 


\section{New bump-bonding technique}

'Standard' eutectic solder ( $\mathrm{Sn} / \mathrm{Pb} 60 / 40)$ melts during

1. high T curing of glue (non-outgassing) used for packaging at $400^{\circ} \mathrm{C}$

2. high temp processing of HPD at $300^{\circ} \mathrm{C}$ (bake-out to remove contaminants)

$\Rightarrow$ Weak bumps (electrically and mechanically)

VTT, Finland: bump-bonding recipe using solder with high melting-point (SnPb 10/90)

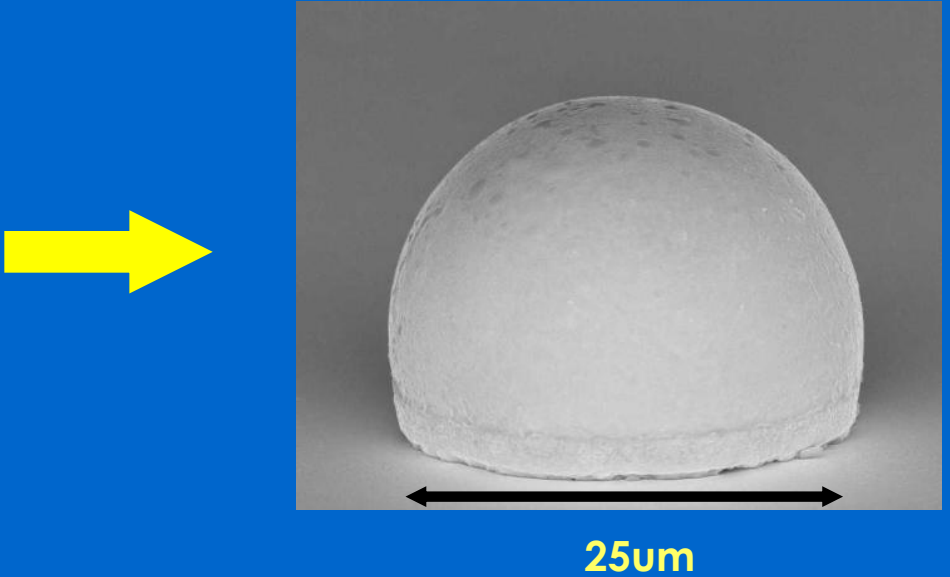

Long programme of tests (bake-outs, SEM analysis, pull-strength tests, prototype HPDs) has successfully proven the bump reliability

VTT producing assemblies with $>99 \%$ good bumps

K.Wyllie 


\section{Interface to HPD: LO board}

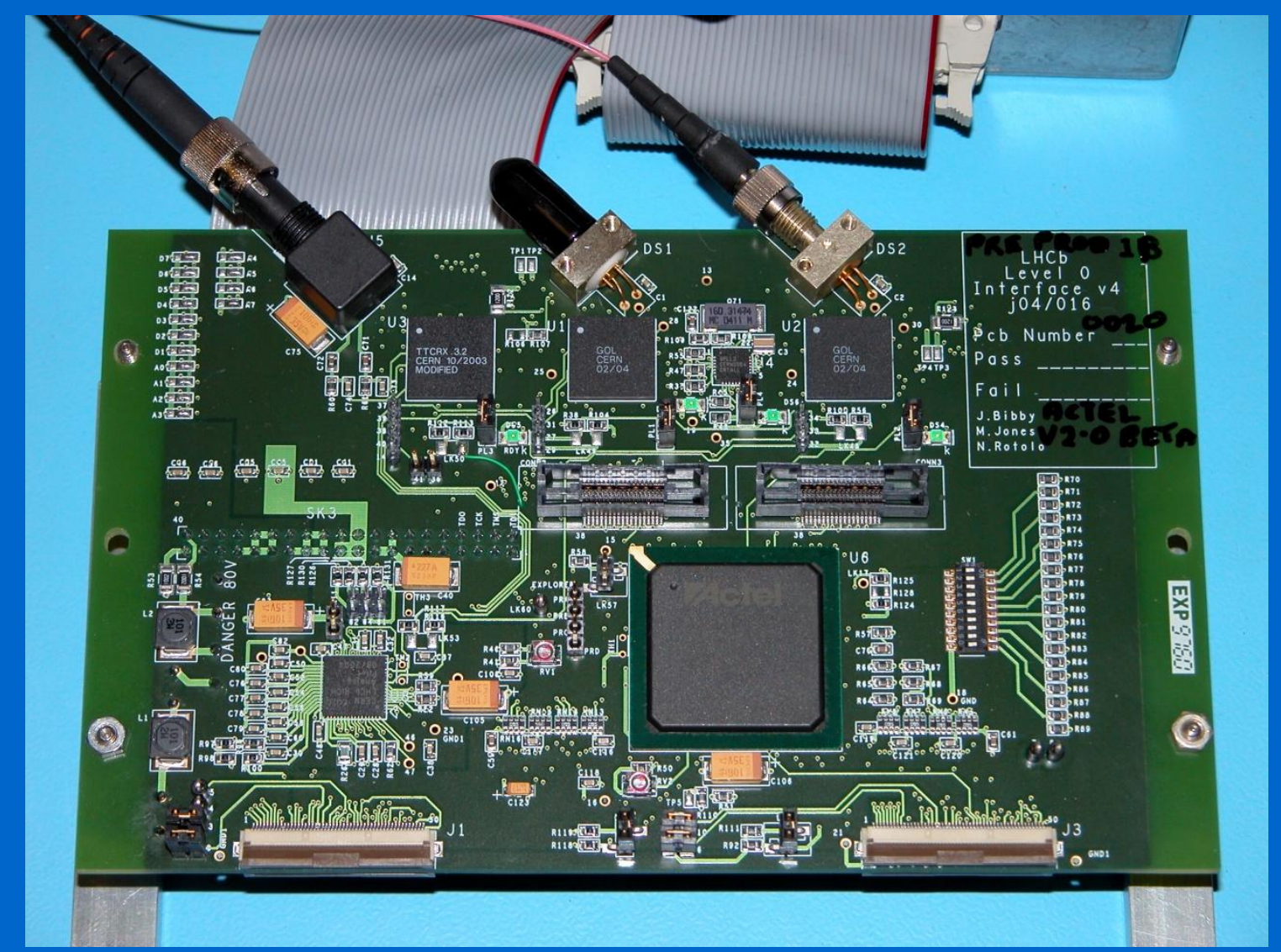

Designed using radiation-tolerant components

Data-formatting by fuse-based gate array

ADCs for temperature monitoring

High speed optical data links:

Each fibre transmits at $1.6 \mathrm{Gbit} / \mathrm{s}$ Full system $=800 \mathrm{Gbit} / \mathrm{s}$ 


\section{High Voltage Distribution}

High Voltage (20kV) boards for dividers, filters, monitoring
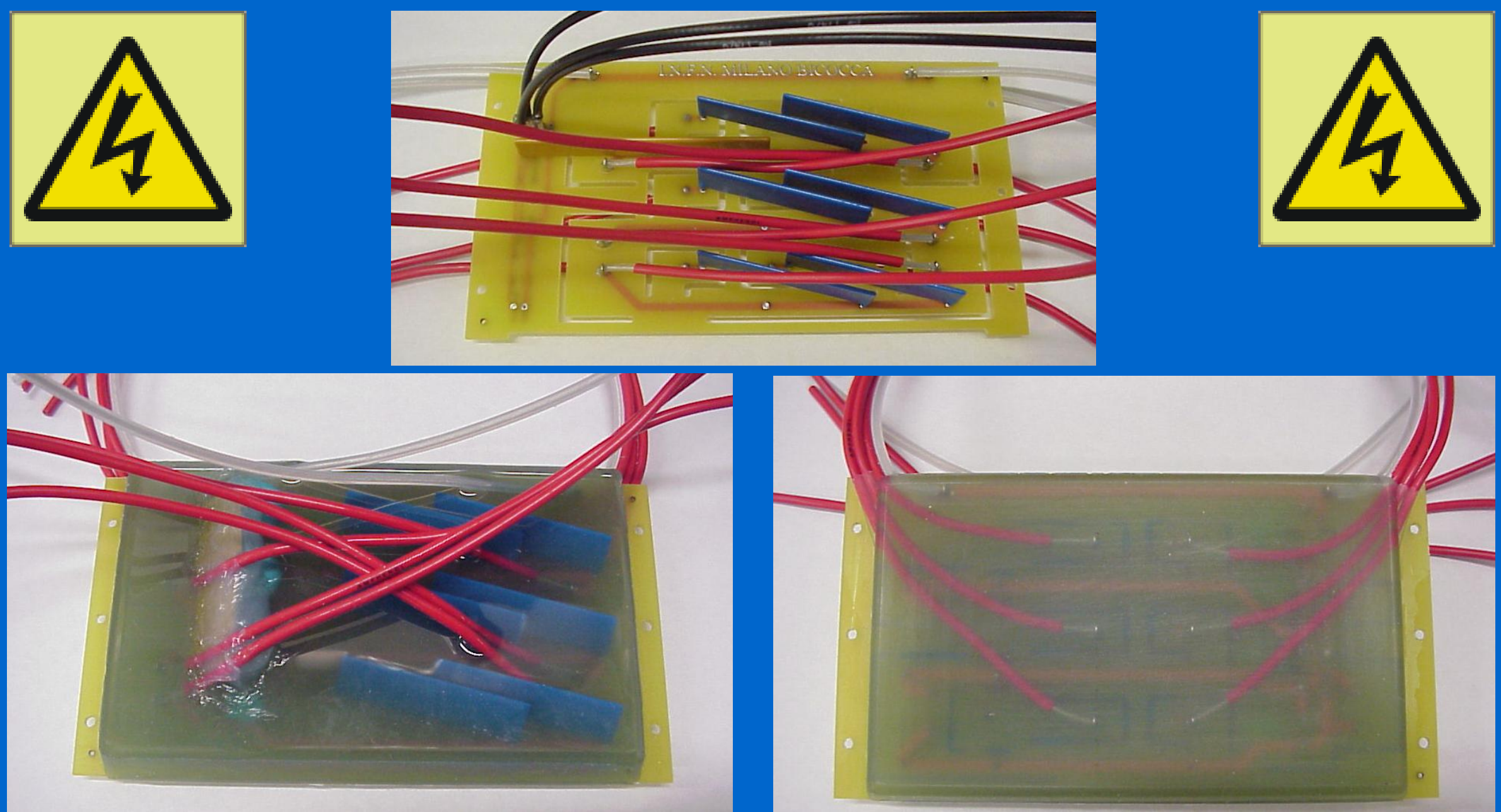

Board after cleaning \& encapsulation in silicone rubber

K.Wyllie

13

Beaune meeting 2005 


\section{Prototype readout electronics system}

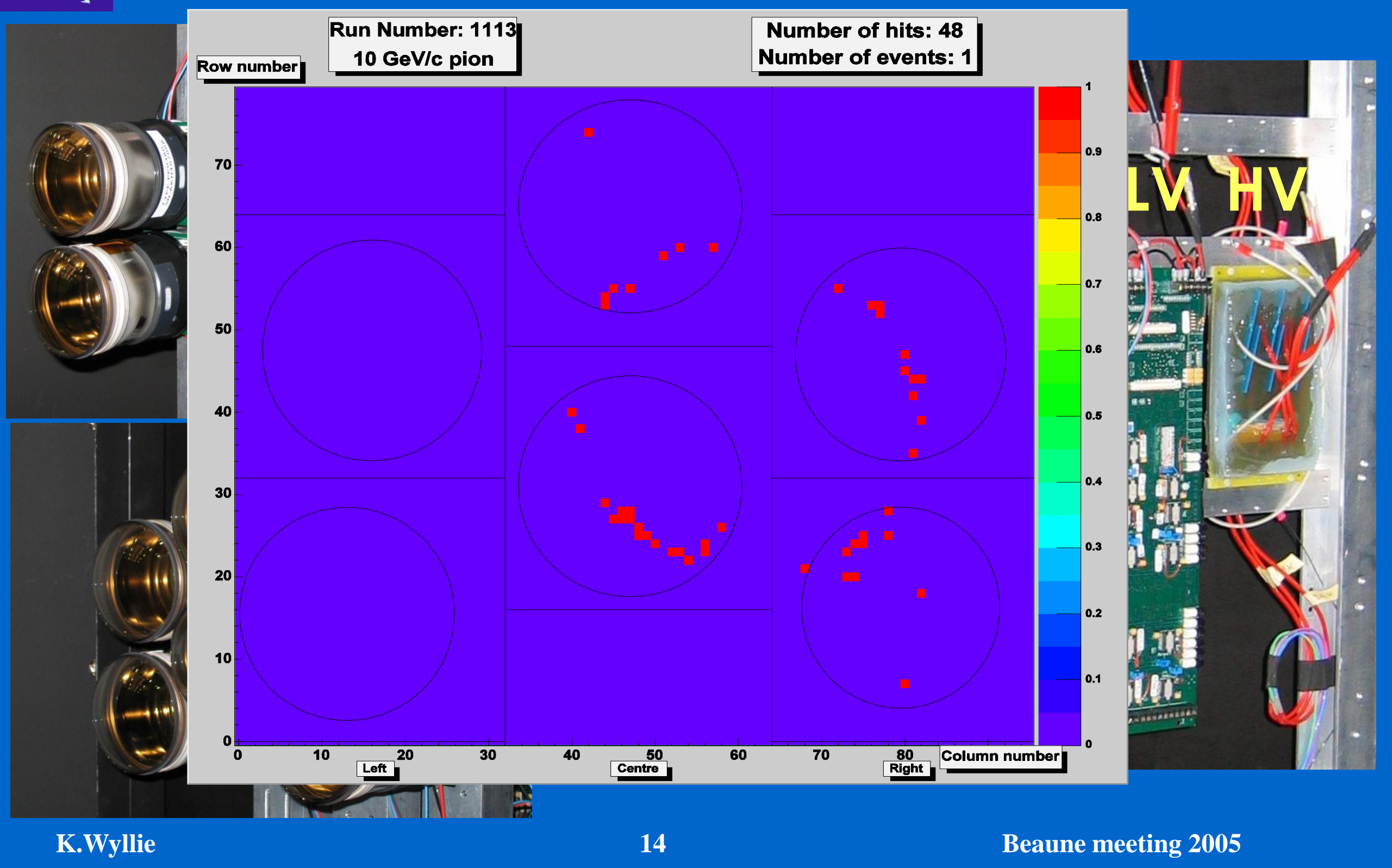




\section{Conclusions}

Pixel chip designed for specific HPD application

New bump-bonding technique developed for HPD compatibility

Specific readout electronics boards designed for data transmission \& power distribution

Electronics in production for large system of 500 HPDs 


\section{Spare slides}




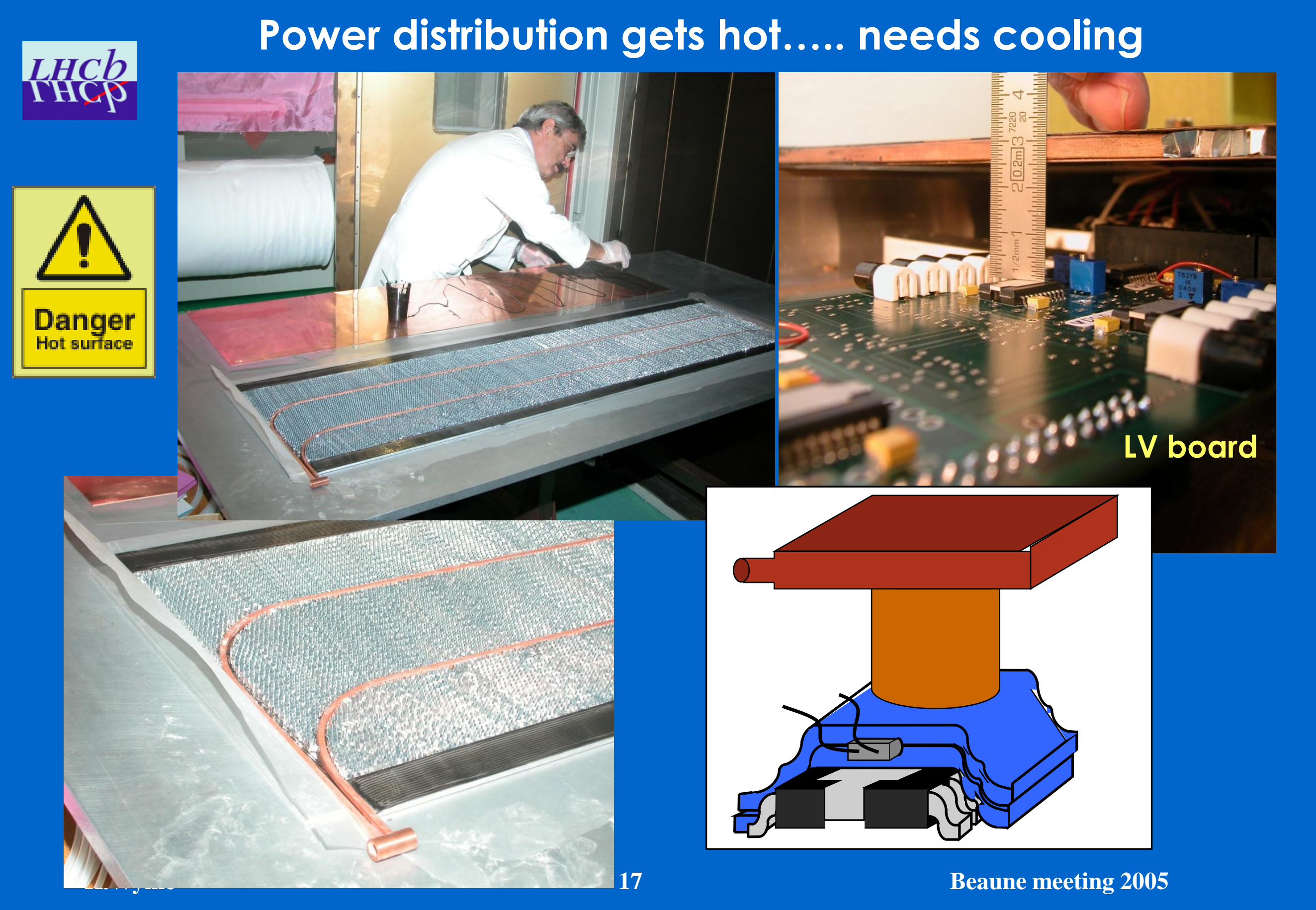




\section{Pixel chip design - LHCBPIX1}

Production run of 48 wafers received beginning 2004

Fully tested ( 3400 chips) on probe-station \& good chip selected according to criteria:

-Threshold \& noise calibration in spec

- Configuration \& power supply tests

- Correct operation at $45 \mathrm{MHz}$

Yield of $55 \% \Rightarrow 3$ times the number of HPDs

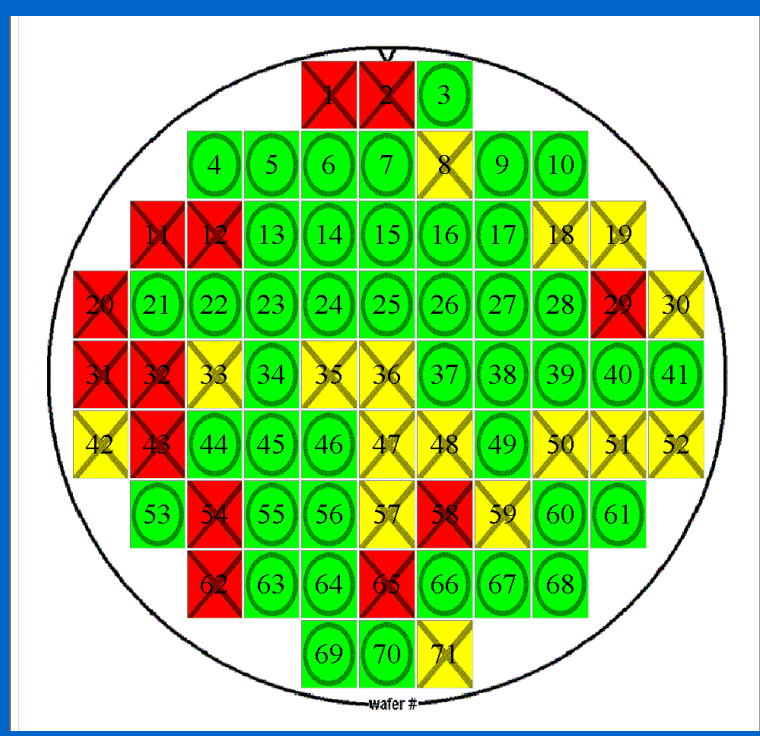

Wafer maps provided for bump-bonding at VTT 

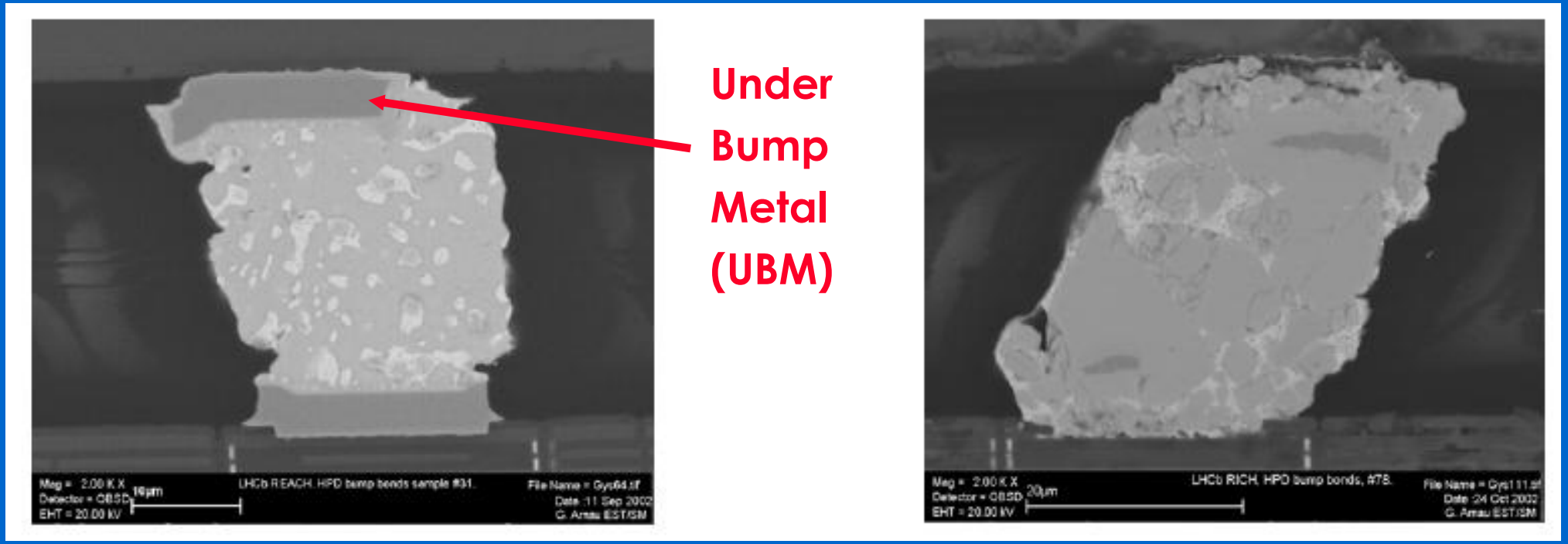

Sensor

10/90 SnPb before bake

10/90 SnPb after bake
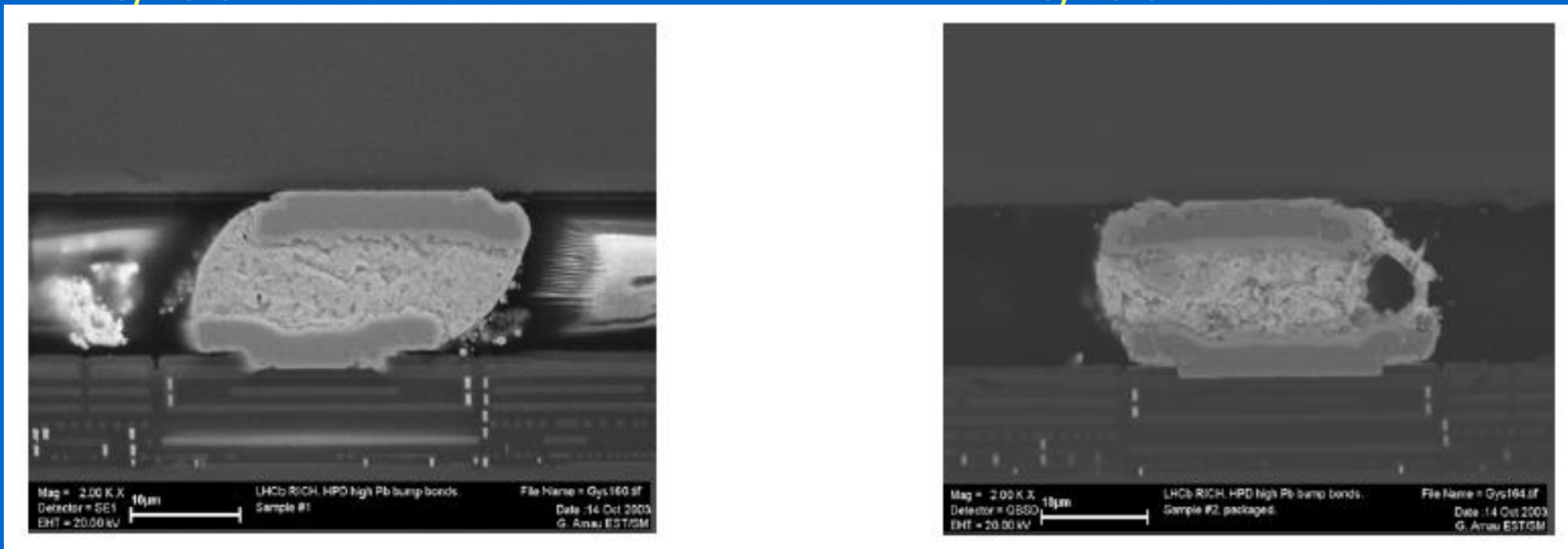

Chip

K.Wyllie

Beaune meeting 2005 


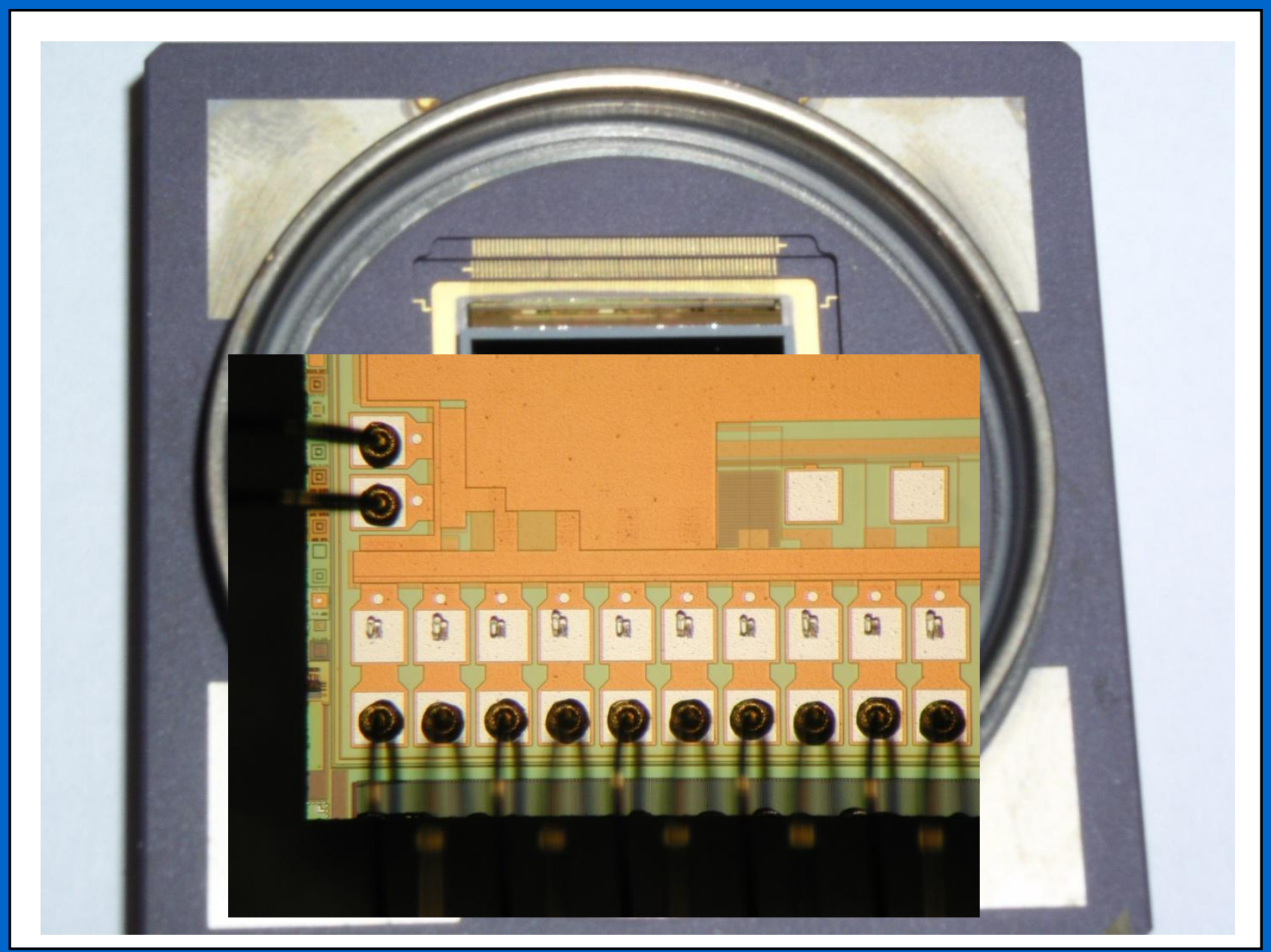

Ceramic carrier produced by Kyocera, Japan

- Good thermal conduction

K.Wyllie 